\title{
Die Ableitung olfaktorisch evozierter Potenziale in Patienten mit limbischer Enzephalitis
}

\section{Olfactory Evoked Potentials in Patients with Limbic Encephalitis}

Autoren

Felix A. Schmidt ${ }^{1}$, Lutz Harms ${ }^{1}$, Harald Prüss ${ }^{1,}$, Rohat Geran $^{1}$, Heidi Olze ${ }^{3}$, Matthew B. Maas ${ }^{4}$, Florian C. Uecker ${ }^{3}$

Institute

1 Klinik für Neurologie, Charité - Universitätsmedizin Berlin, Berlin

2 Deutsches Zentrum für Neurodegenerative Erkrankungen (DZNE), Berlin

3 Klinik für Hals-, Nasen- und Ohrenheilkunde, Charité - Universitätsmedizin Berlin, Berlin

4 Department of Neurology, Northwestern University, Chicago, Illinois, USA

Schlüsselwörter

limbische Enzephalitis, Olfaktorisch Evozierte Potenziale, Riechtestung, Riechstörung

Key words

limbic encephalitis, olfactory evoked potentials, olfactory testing, olfactory dysfunction

Bibliografie

DOI https://doi.org/10.1055/s-0043-124360

Online-Publikation: 14.3.2018

Klin Neurophysiol 2019; 50: 11-16

(c) Georg Thieme Verlag KG Stuttgart · New York

ISSN 1434-0275

Korrespondenzadresse

Dr. med. Felix A. Schmidt

Klinik für Neurologie

Charité - Universitätsmedizin Berlin

Charitéplatz 1

10117 Berlin

felix.schmidt@charite.de

\section{ZUSAMMENFASSUNG}

Ziel der Studie In dieser Studie wurde erstmals das Riechvermögen bei Patienten mit limbischer Enzephalitis (LE) mittels olfaktorisch evozierter Potenziale (OERP) untersucht.

Methodik Bei 19 LE Patienten (9 weibliche, 10 männliche Patienten, mittleres Alter 47 Jahre) und 19 gesunden Kotrollprobanden (GK) wurde das Riechvermögen mittels psychophysischer Schwellen-Diskrimination-Identifikations-Testung (SDI) und mittels OERPs bestimmt.
Ergebnisse 10 LE Pat. (53\%) hatten eine Hyposmie, 2 Pat. (11\%) eine funktionelle Anosmie und 7 Pat. (36\%) waren normosmisch. Der SDI-Mittelwert \pm Standardabweichung betrug bei den LE-Patienten 27,3 $\pm 6,7$; bei den GK 34,7 $\pm 2,2$. Der SDIWert der LE Patienten war im Vergleich zu den GK signifikant vermindert $(p<0,0001)$. Alle untersuchten Patienten mit eingeschränktem Riechvermögen zeigten pathologische OERPs. Schlussfolgerung Riechstörungen konnten gehäuft bei LE Patienten nachgewiesen werden. Möglicherweise sind hierfür strukturelle Schädigungen des limbischen Systems, das an der Weiterverarbeitung von olfaktorischen Informationen beteiligt ist, ursächlich. Die Ableitung von OERPs erwies sich als geeignetes objektives Untersuchungsverfahren zur Detektion von Riechstörungen bei LE Patienten.

\section{ABSTRACT}

Objective Limbic encephalitis (LE) refers to an inflammatory process localized to structures of the limbic system that causes different neurologic and psychiatric disorders. To our knowledge, this is the first study investigating the olfactory function with olfactory evoked potentials (OEP) of patients with paraneoplastic or autoimmune encephalitis.

Methods 19 LE patients ( 9 female, 10 male, mean age 47 years) and 19 age- and sex-matched healthy controls (HC) were tested. The Threshold Discrimination Identification test (TDI) was used to test orthonasal olfactory function. OEPs were recorded for objective olfactometry.

Results 10 LE patients (53\%) were hyposmic, 2 patients anosmic (11\%) and 7 patients normosmic (36\%). Their mean TDI value \pm standard deviation was $27.3 \pm 6.7$. All LE patients with olfactory dysfunction also showed pathological OEPs. All 19 $\mathrm{HCs}$ were normosmic with a mean TDI value of $34.7 \pm 2.2$. There was a significant reduction of the LE patients' TDI score compared to the $\mathrm{HC}(\mathrm{p}<0.0001)$.

Conclusions Olfactory dysfunction seems to be a frequent symptom in LE patients. This might be due to structural changes of the limbic system. OEPs were a suitable method to detect olfactory dysfunction in these patients. 


\section{Einleitung}

Die limbische Enzephalitis (LE) ist eine Autoimmunerkrankung, die mit einer Entzündungsreaktion des limbischen Systems durch pathogene Autoantikörper gegen neuronale Oberflächenstrukturen oder onkoneuronale Antikörper einhergeht [1]. Im tertiären olfaktorischen Kortex, der Teil des limbischen Systems ist, findet u. a. die Weiterverarbeitung der Riech- und Geschmackseindrücke statt $[2,3]$. Bei anderen Autoimmunerkrankungen wie der Multiplen Sklerose und der Antikörper-vermittelten Neuromyelitis Optica Spektrum Erkrankung konnten Riechstörungen nachgewiesen werden [4-6]. Die Diagnostik von Riechstörungen auf diesem Gebiet gewinnt zunehmend an Bedeutung [7]. Eine Riechminderung ist ein Kardinalsymptom bei verschiedenen neurodegenerativen $\mathrm{Er}$ krankungen wie bspw. der Parkinson-Krankheit, und kündigt diese häufig als Frühsymptom an [8]. Die Ableitung von olfaktorisch evozierten Potenzialen (OERP) ist eine objektive Methode zur Untersuchung des Geruchssinns [9]. Dabei wird die kortikale elektrische Aktivität auf die Stimulation mit einem Duftstoff gemessen [10].

Im Rahmen dieser Studie sollte untersucht werden, ob bei LE Patienten durch eine strukturelle Schädigung von Hirnarealen inklusive des limbischen Systems vermehrt Riechstörungen verursacht werden. Nach unserem Kenntnisstand gibt es bisher keine Studie, die das Riechvermögen bei LE Patienten mittels OERPs untersucht hat.

\section{Material und Methoden}

\section{Patienten}

Die Rekrutierung der Patienten erfolgte von der neurologischen Normalstation sowie aus der Sprechstunde für Enzephalitis \& Paraneoplasien der Hochschulambulanz der Charité - Universitätsmedizin Berlin, Campus Mitte, Berlin. Der Studieneinschluss erfolgte nach neurologischer und HNO-ärztlicher Untersuchung. Einschlusskriterien waren die Diagnose einer limbischen Enzephalitis [11] sowie ein Alter von mind. 18 und unter 65 Jahren. Patienten mit mittelgradigen bis schweren kognitiven Defiziten wurden mittels des Mini-Mental-Status Testes ausgeschlossen, da kognitive Einbußen Einfluss auf die Durchführung der subjektiven Riechtestung haben können. Patienten mit einer Depression wurden mittels des BecksDepressions-Inventars ausgeschlossen, da Depressionen das Riechvermögen beeinflussen können. Mittels der Modifizierten Ranking Skala wurde das Ausmaß der körperlichen Beeinträchtigung erfasst um Patienten auszuschließen, die aufgrund körperlicher Behinderung nicht an der objektiven Olfaktometrie teilnehmen können. In der otolaryngealen Untersuchung wurde die Anatomie der Nasenhaupthöhlen und insbesondere der Riechspalten endoskopisch untersucht. Patienten, deren Geruchssinn durch anatomische Auffälligkeiten beeinträchtigt war, wurden von der Studie ausgeschlossen. Folgende weitere Ausschlusskriterien wurden mittels zweier spezieller Fragebögen erfasst: Schwangerschaft, Erkrankungen, die das Riechvermögen beeinträchtigen (posttraumatische Anosmie, sinunasale Erkrankungen, Existenz oder Therapie eines Tumors im Bereich des ersten Hirnnervens, erfolgte Strahlentherapie oder Chemotherapie im Bereich des ersten Hirnnervens, Parkinson Erkrankung, Alzheimer-Krankheit, Depression). Für jeden Patienten wurde ein in Geschlecht und Alter ( \pm 1 Jahr) übereinstimmender gesunder Kontrollproband rekrutiert.
Es konnten 19 LE Patienten und 19 Kontrollprobanden in die Studie eingeschlossen werden. Der Altersmittelwert der Patienten ( 9 weiblich, 10 männlich) betrug 46,8 $\pm 2,0$ Jahre. Es zeigte sich hinsichtlich des Alters bei Frauen (43,9 $\pm 22,2$ Jahre) und Männern $(49,4 \pm 18,5$ Jahre) kein signifikanter Unterschied $(p=0,575)$. Die mittlere Krankheitsdauer betrug 2,0 \pm 1, 1 Jahre. In der serologischen Antikörpertestung ließen sich bei 4 Patienten NMDA-Rezeptor-Ak, 3 Patienten GAD-Ak, 3 Patienten LGI1-Ak, 1 Patienten Caspr2-Ak, 1 Patienten Hu-Ak, 1 Patient DPPX-Ak, 1 Patient CV2Ak, 1 Patient mGluR5-Ak und 2 Patienten mit VGCC-Ak nachweisen. 2 Patienten waren seronegativ getestet und erfüllten klinisch die Kriterien einer LE (11). Sechs Patienten hatten im Krankheitsverlauf epileptische Anfälle und 4 Patienten entwickelten eine Polyneuropathie. Bei 4 Patienten wurde ein paraneoplastischer Tumor diagnostiziert (2x Kleinzelliges Bronchialkarzinom, 1x Teratom, 1x Ovarialkarzinom). Als Immuntherapie erhielten 10 Patienten Rituximab sowie 5 Patienten Plasmapherese-Behandlungen, 2 Patienten intravenöse Immunglobuline (IVIG) und ein Patient eine Kortisonpulstherapie in regelmäßigen Abständen. Ein Patient erhielt keine medikamentöse Therapie.

Ein positives Ethikvotum der Ethikkommission der Charité - Universitätsmedizin liegt vor. Die Patienten und gesunden Kontrollprobanden gaben ihr schriftliches Einverständnis, um an der Studie teilnehmen zu können.

\section{Orthonasale Olfaktometrie}

Das Riechvermögen wurde mithilfe des 3-teiligen, aus 112 Riechstiften bestehenden, standardisierten SDI-Testes (Schwellen-, Diskriminations- und Identifikationstestung, Burghart Messtechnik $\mathrm{GmbH}$, Deutschland) evaluiert. Der Schwellentest dient zur Bestimmung der Geruchswahrnehmungsschwelle und umfasst eine 16fache Verdünnungsreihe, der Diskriminationstest prüfte durch überschwellige Reizung mit 2 gleichen Düften die Fähigkeit zur Unterscheidung von Gerüchen. Im Identifikationstest müssen verschiedene Alltagsgerüche (Kaffee, Fisch, Nelke usw.) identifiziert werden. Unter Addition der 3 Werte entsteht der SDI-Wert. Ein SDIWert von $<16$ bedeutet eine funktionelle Anosmie, $\geq 16$ und $\leq 30,5$ Punkte eine Hyposmie, und über 30,5 Punkten eine Normosmie [12].

\section{Objektive Olfaktometrie}

Zur objektiven Riechprüfung wurden OERP's abgeleitet. Die Olfaktoriusreizstoffe Phenylethylalkohol (PEA) und Schwefelwasserstoff $\left(\mathrm{H}_{2} \mathrm{~S}\right)$ wurden mittels Olfaktometer (OM 2/S Olfaktometer, Burghart Messtechnik GmbH, Deutschland) angeboten ( $>$ Abb. 1). Zur trigeminalen Reizung des Riechepithels wurde Kohlenstoffdioxid $\left(\mathrm{CO}_{2}\right)$ verwendet. Es wurden jeweils 6 verschiedene Testungen nacheinander (jeder der 3 Bedingungen in jeweils seitengetrennter Messung) durchgeführt.

Die Reizdarbietung durch einen in der Nasenöffnung des Patienten fixierten Riechschlauches bei einem konstanten Luftfluss von 7,5 Liter/Minute, einer Lufttemperatur von $37^{\circ} \mathrm{C}$ und einer relativen Luftfeuchte von $70 \%$ gewährleistete eine artefaktfreie Testung. Es wurden verschiedene Reizintensitäten eines jeden Duftstoffes jeweils der rechten und linken Nasenöffnung bei einem randomisierten Reizintervall von 30-45 s mit einer Reizdauer von 200ms angeboten ( $\triangleright$ Abb. 2). Vigilanzstabilisierung des Patienten mittels 
eines einfachen Videospiels sowie eine akustische Abschirmung optimierten die Ableitung der evozierten Potenziale (EP) im Elektroenzephalogramm (EEG). Die Ableitung der EPs erfolgte von den Elektrodenpositionen Fz, Cz und Pz monopolar gegen A1.

Ein OERP ist mit den charakteristischen Peaks P1, N1 und P2 zu erkennen ( $\mathbf{A} \mathbf{b} \mathbf{b}$. $\mathbf{3}$ ). Dabei gelten die Bezeichnungen für $\mathrm{P}=$ positiver Ausschlag und $\mathrm{N}=$ negativer Ausschlag. Die OERPs wurden nach Mittelung von 10 Einzelaufnahmen hinsichtlich ihrer hauptsächlichen Negativität (N1) mit einer Latenz von 200-700 ms und der hauptsächlichen Positivität (P2) mit einer Latenz von 300800 ms vermessen [13]. Die Aufzeichnung der Potenziale erfolgte nicht kontinuierlich, sondern begann 512 ms vor Beginn des Triggers bzw. Reizes und endet 1500 ms nach Beginn des Triggers. Die identifizierten Peaks N1 und P2 wurden in jeder Ableitung mit ihrer jeweiligen Latenzzeit und Amplitudenhöhe ausgemessen. Entscheidend für den positiven Nachweis eines OERPS ist das Vorhandensein der jeweiligen Peaks innerhalb der oben erwähnten Latenzzeiten. Die Auswertung erfolgte nach den Leitlinien der Arbeitsgemeinschaft Olfaktologie und Gustologie der deutschen HNO-Gesellschaft [14].

\section{Mini-Mental-Status-Test (MMST)}

Der Mini-Mental-Status-Test ist ein Screeningverfahren zur Feststellung von Demenzen. Die Scala reicht von 0-30 Punkten. Werte von unter 25 Punkten weisen auf eine kognitive Beeinträchtigung hin [15]. Für unsere Studie wurde eine Gesamtpunktzahl von mind. 25 Punkten als Einschlusskriterium festgelegt.

\section{Becks-Depressions-Inventar (BDI)}

Das Becks-Depressions-Inventar ist ein Selbstbeurteilungsverfahren zur Erfassung der Schwere einer depressiven Symptomatik $[16,17]$. Bei dem Fragebogen, bestehend aus 21 Fragen, können maximal 63 Punkte erreicht werden. Werte zwischen 0 und 8 Punkten sprechen für keine, zwischen 9 und 13 für ein minimale Depression, zwischen 14 und 19 für eine milde, zwischen 20 und 28 für

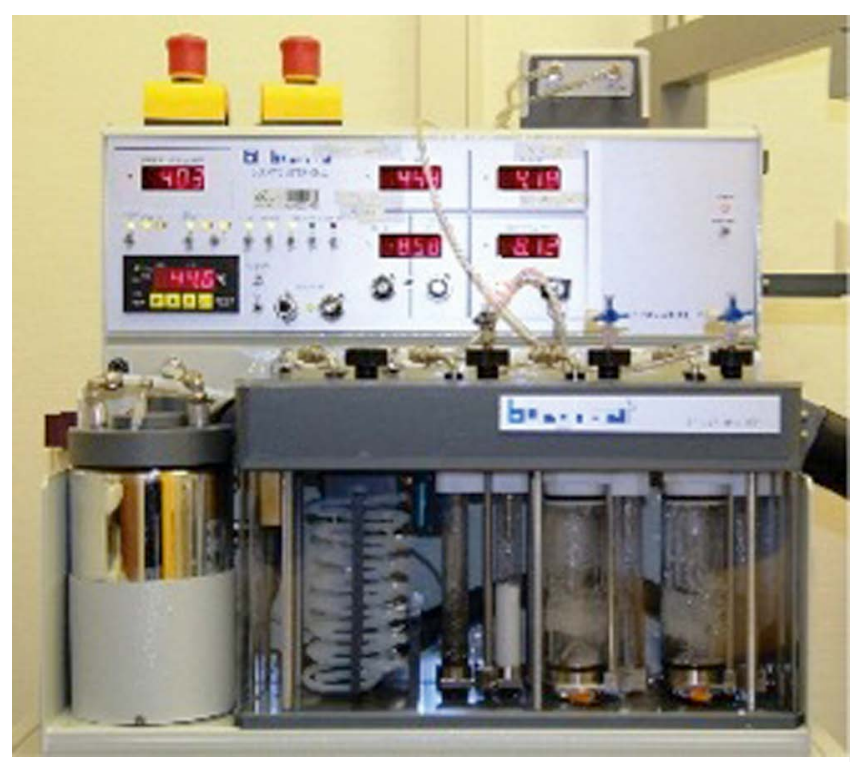

Abb. 1 verwendetes Olfaktometer (OM 2/S Olfaktometer, Burghart Messtechnik GmbH, Wedel, Deutschland). eine mittlere und zwischen 29 und 63 für eine starke Depression. Angepasst an das Patientengut unserer Studie wurde als Einschlusskriterium ein Punktwert von unter 15 Punkten festgelegt.

\section{Modifizierte Ranking-Skala (MRS)}

Die MRS ist ein standardisiertes Messinstrument, welche das Ausmaß der körperlichen Behinderung angibt. Sie ist ein etabliertes Maß für die Beschreibung der neurologischen Beeinträchtigung in klinischen Studien [18]. Die Skala reicht von „Keine Symptome“ (0 Punkte) bis zu „Tod“ (6 Punkte). Wir setzten als Einschlusskriterium eine Punktzahl von< 4 voraus, um an der OERP-Untersuchung teilnehmen zu können.

\section{Statistik}

Die statistische Auswertung erfolgte mit SPSS 22 (SPSS Statistics for Windows, Version 22.0. New York, IBM). Grafen wurden mit GrafPad Prism 7 erstellt (GrafPad Software Inc., La Jola, USA). Um die Zielgrößen miteinander zu vergleichen und auf Unterschiede

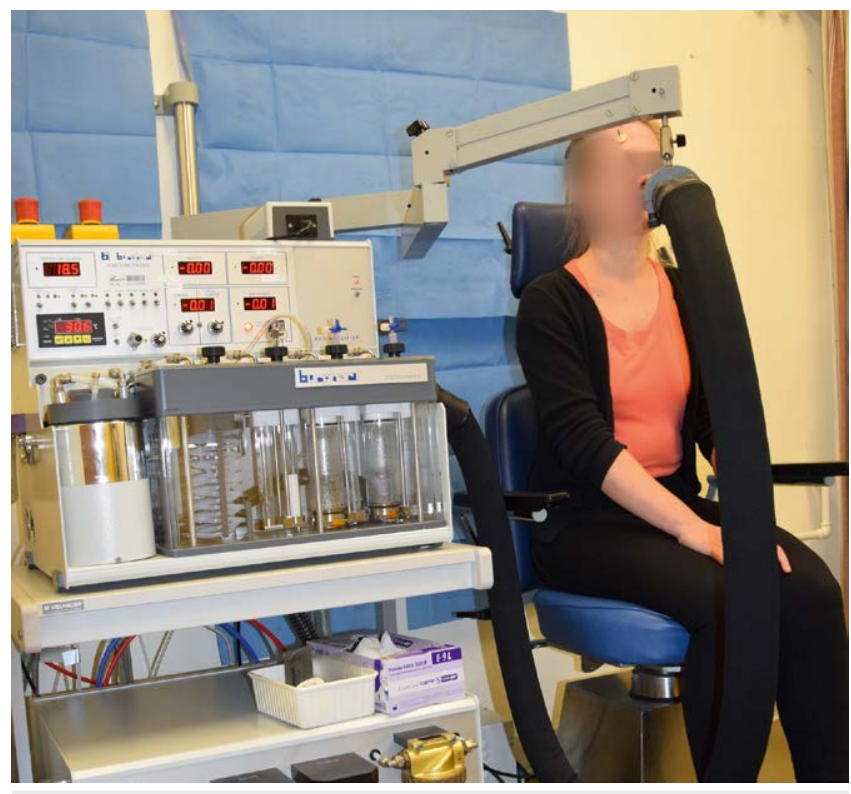

- Abb. 2 Versuchsaufbau Olfaktometer. Gesunde Probandin exemplarisch bei der Messung. Links im Bild das Olfaktometer. Die Duftstoffe werden über den Riechschlauch in die Nasenöffnung der Probandin appliziert.

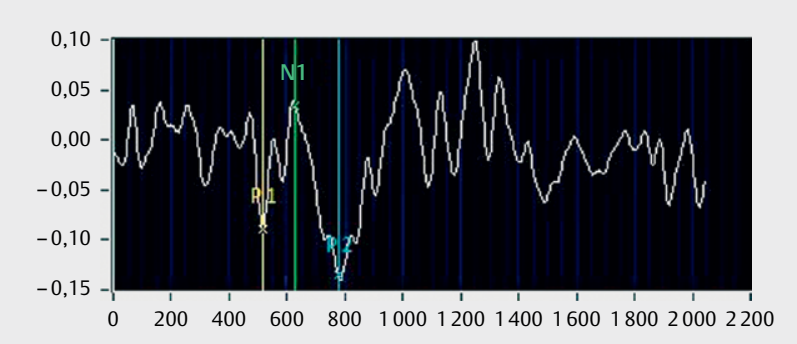

Abb. 3 Abbildung einer OERP-Kurve. Abgebildet ist ein OERP, auf der $x$-Achse ist die Latenzzeit in ms aufgetragen, auf der $y$-Achse ist die OERP-Amplitude in $\mu \mathrm{V}$ angegeben. Die charakteristischen OERPAmplituden-Peaks sind mit P1, N1 und P2 gekennzeichnet. 
- Tab. 1 Demografische und klinische Daten von LE Patienten und gesunden Kontrollen.

\begin{tabular}{|l|c|c|}
\hline & LE (n= 19) & GK (n=19) \\
\hline Alter (in Jahren) & & \\
\hline Mittelwert \pm SA & $46,8 \pm 19,9$ & $47,8 \pm 18,7$ \\
\hline $\begin{array}{l}\text { Geschlecht } \\
\text { weiblich/männlich }\end{array}$ & $9 / 10$ & $9 / 10$ \\
\hline Krankheitsdauer & & \\
\hline Mittelwert \pm SA & $2,0 \pm 1,1$ & - \\
\hline Modifizierte Ranking Skala & & - \\
\hline Mittelwert \pm SA & $1,9 \pm 1,3$ & - \\
\hline Becks Depressions Inventar & & \\
\hline $\begin{array}{l}\text { Mittelwert } \pm S A \\
\text { Mini Mental Status Test }\end{array}$ & $8,8 \pm 7,5$ & - \\
\hline Mittelwert \pm SA & $28,2 \pm 1,3$ & - \\
\hline Antikörpernachweis & & \\
\hline ja/nein & $17 / 2$ & - \\
\hline Immuntherapie & & \\
\hline ja/nein & $18 / 1$ & \\
\hline $\begin{array}{l}\text { GK= gesunde Kontrollen, LE = limbische Enzephalitis, SA = Standard- } \\
\text { Abweichung }\end{array}$ & \\
\hline
\end{tabular}

> Tab. 2 Ergebnisse der subjektiven und objektiven Riechtestung.

\begin{tabular}{|c|c|c|c|}
\hline & $\begin{array}{c}\text { LE } \\
\text { Patienten }\end{array}$ & GK & $\begin{array}{c}\text { p-Wert } \\
\text { (Unterschied } \\
\text { LE vs GK) }\end{array}$ \\
\hline \multicolumn{4}{|l|}{ Subjektive Olfaktometrie } \\
\hline SDI & $27,3 \pm 6,7$ & $34,7 \pm 2,2$ & $<0,0001$ \\
\hline$S$ & $6,2 \pm 3,7$ & $7,8 \pm 1,3$ & 0,182 \\
\hline D & $10,0 \pm 2,9$ & $12,2 \pm 1,2$ & $<0,001$ \\
\hline 1 & $11,1 \pm 2,3$ & $14,7 \pm 0,7$ & $<0,0001$ \\
\hline \multicolumn{4}{|l|}{ Objektive Olfaktometrie } \\
\hline Pathologische OERPs (ja/nein) & $12 / 7$ & - & - \\
\hline \multicolumn{4}{|c|}{$\begin{array}{l}\text { GK = gesunde Kontrollen, LE = limbische Enzephalitis, OERP = olfakto- } \\
\text { risch evoziertes Potenzial, SDI = Schwellen-, Diskriminations- und } \\
\text { Identifikationstest. Die SDI-Ergebnisse sind als Mittelwert } \pm \text { Standard- } \\
\text { abweichung dargestellt }\end{array}$} \\
\hline
\end{tabular}

zwischen den Werten zu prüfen, wurden für normalverteilte Variablen der t-Test und für nicht normalverteilte Variablen der MannWhitney-U-Test verwendet. Das Signifikanzniveau wurde auf $p=0,05$ festgelegt. Zusammenhänge zwischen den einzelnen Werten wurden mittels Spearman-Korrelation untersucht.

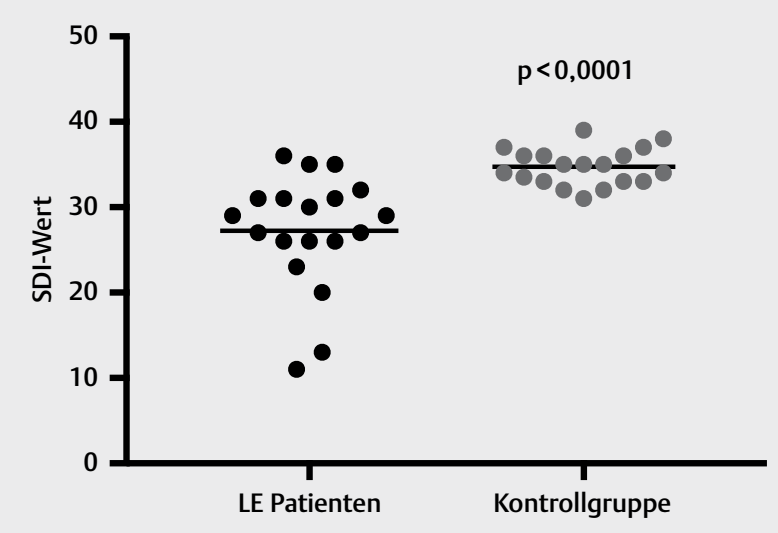

Abb. 4 Vergleich der SDI-Testung von LE Patienten und Kontrollen. Bei Patienten mit LE war signifikant häufiger eine Riechstörung nachweisbar. Die horizontalen Linien markieren den jeweiligen Mittelwert. LE = Limbische Enzephalitis, SDI-Test=Schwellen-, Diskriminations- und Identifikationstest.

\section{Ergebnisse}

Die demografischen und klinischen Daten sind in > Tab. 1, die Ergebnisse der Riechtestung in $>$ Tab. 2 dargestellt.

Im SDI-Test hatten 10 der LE Patienten eine Hyposmie (53\%), 2 Patienten eine Anosmie (11\%) und 7 Patienten (36\%) zeigten eine uneingeschränkte Riechfunktion. Der SDI Mittelwert ( \pm Standardabweichung) der LE Patienten lag bei 27,3 ( $\pm 6,7)$, bei den GK bei 34,7 $\pm 2,2$ Punkten. Es zeigte sich ein signifikanter Unterschied $(p<0,0001)$ zwischen LE Patienten und GK (s. - Abb. 4). In den Einzeltestungen zeigte sich im Diskriminationstest $(p<0,001)$ und im Identifikationstest $(p<0,0001)$ ein signifikanter Unterschied zwischen LE Patienten und GK, jedoch nicht beim Schwellentest $(p=0,182$, > Tab. 2). Es gab bei den LE Patienten beim SDI-Testergebnis keinen signifikanten Unterschied zwischen Männern $(28,8 \pm 5,1$ Punkte) und Frauen (25,6 $\pm 8,2$ Punkte) $(p=0,310)$. Die Identifikationstestung ist durch die einfache Durchführbarkeit und einen kurzen zeitlichen Aufwand (etwa 10 min) charakterisiert. Spezifität und positiver prädiktiver Wert erreichten bei einem Cutoff-Wert von 12 jeweils $100 \%$ um zwischen LE Patienten und GK zu unterscheiden. Die Sensitivität berechnet sich zu $74 \%$ und der negative prädiktive Wert zu $79 \%$.

Mittels visueller Analogskala (VAS), die von 0 Punkten (kompletter Riechverlust) bis 10 Punkte (normales Riechvermögen) reicht, wurden die LE Patienten um eine Einschätzung ihres Riechvermögens gebeten. Die Patienten mit LE hatten ein VAS von 7,7 $\pm 2,2$ (Mittelwert \pm Standardabweichung). Es zeigte sich keine Korrelation zum im SDI-Test gemessenen Riechvermögen. Die Patienten wurden auch nach qualitativem Fehlriechen befragt: es zeigte sich lediglich ein Patient mit einer Phantosmie und ein Patient (jeweils 5,3\%) mit einer Parosmie in der LE Gruppe bei ansonsten nicht eingeschränktem Riechvermögen (Normosmie) in der GK Gruppe.

Unter Nutzung eines Olfaktometers wurden die 19 LE Patienten getestet und deren OERPs abgeleitet. Alle Patienten mit einer Hyposmie oder Anosmie im SDI-Test zeigten auch in der objektiven Olfaktometrie pathologische OERPs und wurden demnach als Hy- 
posmie klassifiziert. Alle Patienten die im SDI Test eine uneingeschränkte Riechfunktion hatten, zeigten auch in der objektiven Olfaktometrie eine Normosmie ( $\triangleright$ Tab. 2).

\section{Diskussion}

Nach unserem Kenntnisstand wurde in dieser Studie erstmals das Riechvermögen bei LE Patienten mittels OERPs untersucht. Es wurde bei insgesamt $64 \%$ der LE Patienten ein vermindertes Riechvermögen in der subjektiven und objektiven Riechtestung festgestellt. Alle gesunden Kontrollprobanden zeigten ein normales Riechvermögen. Es zeigte sich bei den LE Patienten im Vergleich zur GK eine signifikant verminderte Fähigkeit zur Diskrimination und Identifikation von Gerüchen, jedoch kein signifikanter Unterschied bei der Riechschwellentestung. Es wird vermutet, dass mit der Riechschwellentestung die periphere olfaktorische Funktion erfasst wird, wohingegen die Diskriminierung und Identifikation von Gerüchen eher einer komplexeren Verarbeitung unterliegt und von kognitiven Funktionen geprägt ist [19, 20]. Unsere Studienergebnisse deuten auf eine zentrale Schädigung der Riechbahn im sekundären und tertiären olfaktorischen Kortex hin. Dies könnte durch eine strukturelle Schädigung des tertiären olfaktorischen Kortex begünstigt werden, der Teil des limbischen Systems ist und an der Verarbeitung von olfaktorischen Informationen beteiligt ist.

Die Ableitung von OERPs kommt v. a. bei wissenschaftlichen und gutachterlichen Fragestellungen im Fachbereich der HNO, der Neurologie und der Psychiatrie in Betracht [21]. Sie dient zur Abgrenzung eines Restriechvermögens von einer schweren Hyposmie bzw. Anosmie, wobei sich geringgradige Hyposmien gewöhnlich schwer mittels objektiver Olfaktometrie quantifizieren lassen [22]. Dieser Zusammenhang zeigte sich auch in Studien, in denen OERPs bei Patienten mit neurologischen Krankheitsbildern wie Multipler Sklerose, Parkinson-Erkrankung oder Alzheimerdemenz abgeleitet wurden [7]. In unserer Studie ließen sich jedoch bei allen Patienten mit einer Hyposmie in der psychophysischen Riechtestung auch pathologische OERPs nachweisen. Dies könnte durch die wahrscheinlich zentrale Genese der Riechstörung erklärt werden und sich somit in den EEG Ableitungen deutlicher darstellen.

Es wäre in diesem Zusammenhang interessant, mithilfe von Magnetresonanztomografieuntersuchungen strukturelle Schädigungen der Riechbahn und des limbischen Systems mit bspw. der diffusionsgewichteten Bildgebung zu untersuchen, sowie eine Volumenbestimmung olfaktorischer Strukturen durchzuführen und mit dem Riechvermögen der Patienten zu vergleichen.

Alter und Geschlecht der LE Patienten hatten in unserer Studie keinen Einfluss auf die Riechfunktion. Verschiedenen Studien haben gezeigt, dass es bei der normalen Bevölkerung zu einer Abnahme der Riechleistung im Alter durch Ossifikationen in der Lamina cribrosa $[23,24]$ und einem progredienten Verlust von olfaktorischen Rezeptorneuronen im Riechepithel [25] kommt. In verschiedenen Studien zeigte sich, dass es zu einer kontinuierlich zunehmenden Verlängerung der Latenzen und Abnahme der Amplituden der OERPs im höheren Alter kommt [26-29]. Neben dem Alter werden OERPs auch durch das Geschlecht beeinflusst. Untersuchungen haben gezeigt, dass Frauen im Durchschnitt eine höhere OERPAmplitude aufweisen als Männer sowie eine kürzere P2-Latenzzeit [30-33]. Einige Untersuchungen über den Einfluss des Geschlechts auf die psychophysischen Riechtestungen stellten fest, dass Frauen im SDI Test gegenüber Männern häufiger bessere Ergebnisse erzielten [34]. Andere Untersuchungen konnten dagegen keinen Einfluss des Geschlechts auf SDI-Ergebnissen nachweisen [35]. In unserer Studie zeigte das Geschlecht und Alter keinen Einfluss auf die Riechfunktion. Unsere Ergebnisse unterstützen die Hypothese, dass bei LE Patienten andere Pathomechanismen für das Entstehen von Riechstörungen verantwortlich sind.

Die Selbsteinschätzung des Riechvermögens der LE Patienten war besser als die psychophysisch und objektiv erhobenen Riechtestergebnisse und es zeigte sich keine Korrelation zum SDI-Wert. Die limbische Enzephalitis ist ein heterogenes Krankheitsbild mit variablem anatomischen Schwerpunkt, das sich häufig subakut manifestiert und progredient voranschreitet. Möglicherweise kommt es zu einem Gewöhnungsprozess an eine langsam progrediente Riechminderung bei den Patienten. Dieses Phänomen konnte auch bei Patienten mit Multipler Sklerose beobachtet werden [4].

Der Geruchssinn ist wichtig bei der Wahrnehmung von bspw. Brandgeruch, beim Essen und Trinken und bei der interpersonellen Kommunikation. Riechstörungen können zu einer verminderten Lebensqualität führen [36] und sind mit einem erhöhten Risiko für Depressionen assoziiert [37]. Ihr frühzeitiges Erkennen im Krankheitsverlauf ist umso wichtiger, deshalb würden wir eine Riechtestung bei LE-Patienten im klinischen Alltag empfehlen. Darüber hinaus könnte ein Zusammenhang zwischen dem Riechvermögen und dem Aktivitätsgrad der Erkrankung bestehen. In Einzelfällen könnte der sensibilisierte Patient eine akute Verschlechterung selbst erkennen.

Zusammenfassend ließen sich bei der Mehrheit der LE Patienten Riechstörungen nachweisen und zuverlässig mittels OERPs quantifizieren. Die gewonnenen Erkenntnisse sollen zu einem besseren Verständnis des Krankheitsbildes und zukünftig zu einem frühzeitigeren Erkennen von Riechstörungen bei LE Patienten beitragen. Wir würden den Identifikations-Test als Screening-Test bei V.a. LE empfehlen, da dieser eine hohe Spezifität und Sensitivität zur Differenzierung zwischen LE-Patienten und GK aufwies und einfach und schnell durchführbar ist. Weitere Studien mit einer größeren Anzahl an Patienten sind nötig, um unsere Studienergebnisse zu validieren und mögliche Zusammenhänge zwischen dem Riechvermögen und der Krankheitsaktivität zu untersuchen.

\section{KERNBOTSCHAFT}

Riechstörungen kommen gehäuft bei LE Patienten vor. Die Ableitung von OERPs erwies sich als geeignetes objektives Untersuchungsverfahren zur Detektion von Riechstörungen bei LE Patienten.

Interessenkonflikt

LH has received speaker honoraria from Biogen, Teva, Bayer and Novartis. He serves on the advisory board for Roche, Novartis, Genzyme and has received travel support from Biogen, Reche, Bayer and Serono.

FS has received speaker honoraria from Genzyme for a case presentation (Multiple Sclerosis) and travel support from TEVA for ECTRIMS 2017 conference. 
Literatur

[1] Dalmau J. NMDA receptor encephalitis and other antibody-mediated disorders of the synapse: The 2016 Cotzias Lecture. Neurology 2016; 87: 2471-2482

[2] Lemon CH, Katz DB. The neural processing of taste. BMC Neurosci 2007; 18: 5

[3] Small DM. Central gustatory processing in humans. Adv Otorhinolaryngol. 2006; 63: 191-220

[4] Uecker $\mathrm{FC}$, Olze $\mathrm{H}$, Kunte $\mathrm{H}$ et al. Longitudinal testing of olfactory and gustatory function in patients with multiple sclerosis. PLoS One 2017; 12: e0170492

[5] Schmidt F, Göktas O, Harms L et al. Structural correlates of taste and smell loss in encephalitis disseminata. PloS One 2011; 6: e19702

[6] Schmidt F, Göktas $O$, Jarius S et al. Olfactory dysfunction in patients with neuromyelitis optica. Multiple Sclerosis International 2013; 2013: 654501

[7] Godoy MD, Voegels RL, Pinna Fde R et al. Olfaction in neurologic and neurodegenerative diseases: a literature review. Int Arch Otorhinolaryngol 2015; 19: 176-179

[8] Müller A, Mungersdorf $\mathrm{M}$, Reichmann $\mathrm{H}$ et al. Olfactory function in Parkinsonian syndromes. J Clin Neurosci 2002; 9: 521-524

[9] Hummel T, Klimek L, Welge-Lüssen A et al. Chemosensory evoked potentials for clinical diagnosis of olfactory disorders. HNO 2000; 48: 481-485

[10] Lorig TS. The application of electroencephalographic techniques to the study of human olfaction: a review and tutorial. Int J Psychophysiol 2000; 36: 91-104

[11] Graus F, Titulaer M], Balu R et al. A clinical approach to diagnosis of autoimmune encephalitis. Lancet Neurol. 2016; 15: 391-404

[12] Hummel T, Kobal G, Gudziol H et al. Normative data for the "Sniffin' Sticks" including tests of odor identification, odor discrimination, and olfactory thresholds: an upgrade based on a group of more than 3,000 subjects. Eur Arch Otorhinolaryngol 2007; 264: 237-243

[13] Hummel T, Kobal G. Olfactory event-related potentials. In: Simon SA, Nicolelis MAL.(eds) Methods and frontiers in chemosensory research. Boca Raton, Florida, USA: CRC press; 2001: 429-464

[14] Arbeitsgemeinschaft der Wissenschaftlichen Medizinischen Fachgesellschaften. S2k-Leitlinie. Riech- und Schmeckstörungen. 31.10.2016. URL: http://www.awmf.org/uploads/tx_szleitlinien/017-050l_S2k_ Riech-und-Schmeckstörungen_2017-03

[15] Tombaugh TN, McIntyre NJ. The mini-mental state exmanination: a comprehensive. Journal of the American Geriatrics Society 1992; 40: 922-935

[16] Hautzinger M. The Beck Depression Inventory in clinical practice. Nervenarzt 1991; 62: 689-696

[17] Beck AT, Steer RA. Internal consistencies of the original and revised Beck Depression Inventories. Journal of Clinical Psychology 1984; 40: 1365-1367

[18] Van Swieten J, Koudstaal P, Visser M et al. Interobserver agreement for the assessment of handicap in stroke patients. In: Stroke. 19, Nr. 5 1988; S 604-607
[19] Jones-Gotman M, Zatorre RJ. Olfactory identification deficits in patients with focal cerebral excision. Neuropsychologia 1988; 26 : 387-400

[20] Masaoka Y, Yoshimura N, Inoue M et al. Impairment of odor recognition in Parkinson's disease caused by weak activations of the orbitofrontal cortex. Neurosci Lett 2007; 412: 45-50

[21] Stuck BA, Beule A, Damm M et al. Positionspapier „Die chemosensorische Testung bei der gutachterlichen Abklärung von Riechstörungen“. Laryngo-Rhino-Otol 2014; 93: 327-329

[22] Hummel T, Welge-Lüssen A. Riech- und Schmeckstörungen. 1. Auflage 2009 Stuttgart: Thieme Verlag

[23] Doty RL. The olfactory system and its disorders. Semin Neurol 2009; 29: 74-81

[24] Wilson RS, Yu L, Schneider JA et al. Lewy bodies and olfactory dysfunction in old age. Chem Senses. 2011; 36: 367-373

[25] Liss L, Gomez F. The nature of senile changes of the human olfactory bulb and tract. AMA Arch Otolaryngol 1958; 67: 167-171

[26] Covington JW, Geisler MW, Polich J et al. Normal aging and odor intensity effects on the olfactory event-related potential. Int J Psychophysiol 1999; 32: 205-214

[27] Hummel T, Futschik T, Frasnelli ] et al. Effects of olfactory function, age, and gender on trigeminally mediated sensations: a study based on the lateralization of chemosensory stimuli. Toxicol Lett 2003; 140-141: 273-280

[28] Murphy C, Morgan CD, Geisler MW et al. Olfactory event-related potentials and aging: normative data. Int J Psychophysiol 2000; 36 : 133-145

[29] Doty RL, Kamath V. The influences of age on olfaction: a review. Front Psychol 2014; 5: 20

[30] Ohla K, Lundstrom JN. Sex differences in chemosensation: sensory or emotional? Front Hum Neurosci 2013; 7: 607

[31] Morgan CD, Geisler MW, Covington JW et al. Olfactory P3 in young and older adults. Psychophysiology 1999; 36: 281-287

[32] Olofsson JK, Nordin S. Gender differences in chemosensory perception and event-related potentials. Chem Senses 2004; 29: 629-637

[33] Lundström JN, Frasnelli J, Larsson M et al. Sex differentiated responses to intranasal trigeminal stimuli. Int J Psychophysiol 2005; 57: 181-186

[34] Jacob T], Fraser C, Wang L et al. Psychophysical evaluation of responses to pleasant and mal-odour stimulation in human subjects; adaptation, dose response and gender differences. Int J Psychophysiol 2003; 48: 67-80

[35] Evans W], Cui L, Starr A. Olfactory event-related potentials in normal human subjects: effects of age and gender. Electroencephalogr Clin Neurophysiol 1995; 95: 293-301

[36] Temmel AF, Quint C, Schickinger-Fischer B et al. Characteristics of olfactory disorders in relation to major causes of olfactory loss. Arch Otolaryngol Head Neck Surg 2002; 128: 635-641

[37] Kohli P, Soler ZM, Nguyen SA et al. The association between olfaction and depression: A systematic review. Chem Senses. 2016; 41: 479-486 